\title{
ERROS NA MEDICAÇÃO: ANÁLISE DAS SITUAÇÕES RELATADAS PELOS PROFISSIONAIS DE ENFERMAGEM ${ }^{*}$
}

\author{
MEDICATION ERRORS : ANALYSIS OF SITUATIONS REPORTED BY NURSING STAFF
}

Viviane Tosta de Carvalho ${ }^{1}$ \& Silvia Helena De Bortoli Cassiani ${ }^{2}$

${ }^{1}$ Enfermeira. Mestre pela Escola de Enfermagem de Ribeirão Preto-USP; ${ }^{2}$ Docente do Departamento de Enfermagem Geral e Especializada da Escola de Enfermagem de Ribeirão Preto-USP.

Correspondência: Viviane Tosta de Carvalho. Rua Otto Benz, 523. Nova Ribeirânia. CEP 14096-580 Ribeirão Preto; São Paulo; Brasil. email: vtrbarros@uol.com.br.

CARVALHO VT \& CASSIANI SHB. Erros na medicação: análise das situações relatadas pelos profissionais de enfermagem. Medicina, Ribeirão Preto,33: 322-330, ,jul./set. 2000.

RESUMO: Os erros na medicação são alguns dos indicadores da qualidade de saúde prestada aos pacientes hospitalizados. O objetivo deste estudo foi analisar as situações que conduziram os profissionais de enfermagem aos erros na administração de medicamentos, com base em relatos de erros ocorridos. O local de estudo foi um hospital no interior do Estado de São Paulo. Foram entrevistados sete enfermeiros, um técnico de enfermagem e 23 auxiliares de enfermagem do setor de clínica médica do referido hospital. O referencial metodológico adotado foi uma adaptação da técnica do Incidente Crítico. Da análise foram identificadas 56 situações, agrupadas em quatro categorias: falha no cumprimento de políticas e procedimentos (25), falha no sistema de distribuição e preparo dos medicamentos pela farmácia (15), falha na comunicação (10) e falha no conhecimento (06). Fornecer um ambiente seguro com disponibilidade de recursos humanos e físicos faz-se necessário para a prevenção de futuros erros de medicação, assim como investimentos no conhecimento sobre administração de medicamentos aos profissionais de enfermagem, visando a uma assistência de enfermagem com qualidade.

UNITERMOS: Erros de Medicação. Medicamentos. Enfermagem.

\section{1- INTRODUÇÃO}

Com o advento de inúmeros medicamentos no mercado e o avanço tecnológico da indústria farmacêutica, a administração de medicamentos tornou-se uma tarefa extremamente complexa, requerendo dos profissionais da saúde cada vez mais responsabilidades, conhecimentos farmacológicos, de anatomia e fisiologia e habilidades técnicas.

O enfermeiro, no decorrer da sua formação profissional, adquire conhecimentos específicos que o capacitam a exercer com habilidade a função de administrar o medicamento; sendo assim, é de sua responsabi- lidade o preparo e a administração de medicamentos aos pacientes, em diversas instituições hospitalares.

Entretanto, vários autores têm apontado o escasso conhecimento de farmacologia, fisiologia e anatomia e, conseqüentemente, complicações após a administração de medicamentos injetáveis e a não-observância de procedimentos técnico ${ }^{(1 / 4)}$.

O despreparo dos profissionais na administração de medicamentos tem causado sérias consequiências ao paciente, muitas vezes debilitantes, desencadeando reações indesejadas, como complicações advindas de procedimentos técnicos inadequados ou incorretos decorrentes dos erros na medicação. 
Erro na medicação é definido como:

"qualquer evento previsivel que pode ser causado ou surgir do uso inconveniente ou falta de uma medicação ou causar prejuízo (dano ou injúria) ao paciente, enquanto a medicação está sob o controle dos profissionais da saúde, pacientes ou consumidor. Tais eventos podem estar relacionados à prática profissional, aos produtos para o cuidado à saúde, procedimentos e sistemas, incluindo a prescrição, comunicação da prescrição, rótulo do produto, embalagem e nomenclatura; à composição, à distribuição; à administração; à educação dos enfermeiros e pacientes; à supervisão e uso"(5).

Diversas instituições e pesquisadores, preocupados com a questão dos erros de medicação, têm desenvolvido estratégias para reduzir ou prevenir erros na medicação. Bates et al. ${ }^{(6)}$ afirmam que, em cada 100 pacientes admitidos nos hospitais, $4,8 \%$ apresentam eventos adversos e os erros com a medicação ocorrem mais freqüentemente nos estágios da prescrição médica (56\%), transcrição da prescrição médica (6\%), distribuição do medicamento (4\%) e administração do medicamento (34\%).

Medicamentos administrados erroneamente podem ter efeitos drásticos e resultar em sérios prejuízos ou danos ao paciente. Wolf et al. ${ }^{(7)}$ classificam os danos aos pacientes nessa ordem: lesões do sistema nervoso central, reações de hipersensibilidade, amputação de membros, diminuição da acuidade visual e auditiva, aumento de dor quando há omissão do medicamento e até a morte.

Há, também, conseqüências para o profissional que cometeu o erro. Geralmente, a "culpa" recai sobre o profissional que executou a ação final do processo de administrar medicamentos, mesmo que tenha se iniciado em outros setores. Assim, a enfermagem é a responsável direta, pois está na linha final do sistema, cabendo medidas disciplinares que vão desde uma orientação até a demissão.

Desta forma, cria-se uma subnotificação dos erros, face ao medo das punições. É imprescindível que, na avaliação do erro, os supervisores considerem não só os aspectos técnicos, mas, também, os outros fatores que podem desencadeá-lo.

Fatores relacionados à organização do trabalho, como o acúmulo de atividades, recursos humanos insuficientes e malqualificados, locais desprovidos de materiais, aparelhos e recursos financeiros; fatores ambientais, como planta física inadequada, freqüentes interrupções de outros profissionais durante o preparo da medicação pelo profissional de enfermagem, presença de ruídos, luminosidade são alguns que devem ser considerados nas situações de erros na medicação.

Consideramos, ainda, que, na nossa realidade, os profissionais de enfermagem também assumem dupla jornada de trabalho, com vínculo empregatício em duas ou mais instituições de saúde, o que gera sobrecarga de trabalho, promove a fadiga, estresse e desatenção no ambiente de trabalho e predispõe à ocorrência do erro na medicação.

Portanto, na ocorrência de um erro, o hospital deve iniciar, imediatamente, uma investigação completa, determinando e documentando os detalhes exatos da natureza do acontecido como: horário, pessoal envolvido, turno, tipo de erro e, entre outros aspectos, possíveis riscos nas fases anteriores à execução do erro e o que o desencadeou, o estágio do processo em que o erro ocorreu e possíveis falhas do sistema, a fim de amenizá-lo, evitando que a culpa recaia somente no indivíduo profissional.

Infelizmente, pouco é realizado, nas instituições de saúde brasileiras, para a verificação do motivo do erro. As intervenções adotadas pela chefia frente à ocorrência do erro, em geral, são ações punitivas e individuais de censura, advertências verbais, relatórios, transferência para outro setor e possíveis demissões da instituição. Raramente, treinamentos e reciclagem necessários à prevenção de futuros erros são efetivamente realizados. O indivíduo é penalizado conforme a gravidade e consequiência do erro ao paciente ${ }^{(8)}$.

Dessa maneira, este estudo foi planejado com o objetivo de resposta à seguinte questão: "Em quais situações os erros de medicação ocorrem?". Pretende-se identificar e analisar os erros na medicação de uma instituição hospitalar no interior do Estado de São Paulo a partir dos relatos dos profissionais de enfermagem.

\section{2- MATERIAL E MÉTODO}

\subsection{Local de estudo}

O estudo foi realizado em um hospital no interior do Estado de São Paulo, setor de clínica médica.

\subsection{População e amostra}

A população em estudo e atuante no setor era composta por dez enfermeiros, quatro técnicos e quarenta e seis auxiliares de enfermagem. Foram excluídos os enfermeiros no cargo de chefia. 
A amostra ficou constituída por sete enfermeiros, um técnico de enfermagem e vinte e três auxiliares de enfermagem, haja vista que, do total de enfermeiros, um enfermeiro estava de licença-saúde, um recusou-se a participar do estudo e houve um relato inválido. Do total de quarenta e seis auxiliares de enfermagem alocados, onze recusaram-se a participar do estudo, um estava de licença-saúde, um tinha sido demitido, um transferido para outro setor. Do total de trinta e dois auxiliares de enfermagem entrevistados, oito não recordaram nenhum fato e um apresentou o relato inválido.

Dos quatro técnicos entrevistados: um não recordou nenhum fato, um recusou participação e um apresentou um relato inválido. Consideramos relatos inválidos os relatos incompletos, que não apresentavam as situações que conduziram os profissionais de enfermagem aos erros na administração de medicamentos. Assim, foram realizadas 31 entrevistas com 46 relatos válidos.

\section{3- REFERENCIAL METODOLÓGICO}

\subsection{A técnica do Incidente Crítico}

A técnica do Incidente Crítico é um método indireto de análise que permite o registro de comportamentos específicos, favorecendo observações e avaliações de forma sistematizada.

"Essa técnica consiste de um conjunto de procedimentos para a coleta de observações diretas do comportamento humano, de modo a facilitar sua utilização potencial na solução de problemas práticos e no desenvolvimento de amplos princípios psicológicos, delineando também procedimentos para a coleta de incidentes observados, que apresentam significado especial e para o encontro de critérios sistematicamente definidos" (9).

Incidente é definido como "qualquer atividade humana observável que seja suficientemente completa em si mesma para permitir inferências e previsões a respeito da pessoa que executa o ato" ${ }^{(9)}$. Portanto, o incidente crítico prevê a análise de uma ocorrência crítica que marcou as pessoas.

Neste estudo, utilizamos uma adaptação da técnica do Incidente Crítico já que foram abordados somente os aspectos negativos. Acreditamos que não há aspectos positivos na ocorrência de um erro de medicação.

\subsection{Coleta dos dados. Ética na pesquisa}

O instrumento de coleta de dados constou de um formulário para a obtenção de relatos de erros ocorridos na medicação, de acordo com o objetivo do estudo. $\mathrm{O}$ instrumento foi submetido a um pré-teste com uma (01) enfermeira mestranda, atuante em um hospital privado, um (01) técnico de enfermagem e uma (01) auxiliar de enfermagem, atuantes no hospital em estudo, porém, em outro setor.

Findo o período pre-teste, iniciamos a coleta de dados, nos meses de agosto e setembro de 1999, após aprovação do estudo pelo Comitê de Ética em Pesquisa do Hospital em questão e o consentimento da divisão de enfermagem e da diretoria de enfermagem da unidade.

Após o consentimento informado dos participantes do estudo, as entrevistas foram marcadas e realizadas na própria instituição hospitalar, durante o período de trabalho, e transcorreram, em média, em 15 a 30 minutos. A questão abordada foi: "Pense em alguma coisa negativa, ruim que ocorreu na administração de medicamentos, recentemente, com pacientes da sua unidade. Pense em uma ocorrência que lhe chamou a atenção e resultou em um erro de medicação. Conte-me qual foi a situação?”. As entrevistas foram gravadas em fita cassete e transcritas na íntegra, imediatamente após o seu término. Foram mantidos o sigilo das informações e o anonimato dos entrevistados.

\subsection{Organização dos dados para análise}

Após o término da coleta dos dados, iniciou-se a análise das entrevistas, procedendo-se à leitura dos relatos e identificação das situações. A SITUAÇÃO foi definida como o fato ou circunstância que levou o profissional ao erro.

Os dados foram agrupados por semelhança de conteúdo, totalizando 56 situações agrupadas em quatro categorias. A seguir, apresentaremos a identificação e análise das situações categorizadas.

\section{4- RESULTADOS E DISCUSSÃO}

As situações foram agrupadas em quatro categorias: Falha no cumprimento de políticas e procedimentos, Falha no sistema de distribuição e preparo de medicamento pela farmácia, Falha na comunicação e Falha no conhecimento.

Apresentamos, na Tabela I, as categorias de situações, seus conteúdos e as frequiências em que os erros ocorreram, segundo os profissionais de enfermagem. 


\begin{tabular}{|c|c|c|}
\hline Situações & Conteúdo & Freqüência $N^{o}$ \\
\hline $\begin{array}{l}\text { 1. Falha no cumprimento de } \\
\text { políticas e procedimentos }\end{array}$ & $\begin{array}{l}\text { - Falha na execução da técnica } \\
\text { - Falha na identificação do paciente }\end{array}$ & $\begin{array}{l}15 \\
10\end{array}$ \\
\hline Subtotal & & 25 \\
\hline $\begin{array}{l}\text { 2. Falha no sistema de } \\
\text { distribuição e preparo dos } \\
\text { medicamentos }\end{array}$ & $\begin{array}{l}\text { - Atraso no horário de envio do carro à clínica } \\
\text { - Medicamentos com rótulo e embalagem semelhantes } \\
\text { - Muitos medicamentos no mesmo horário } \\
\text { - } \quad \text { Medicamento enviado com apresentação errada } \\
\text { - } \quad \text { Carro com cartão em casela errada } \\
\text { - } \quad \text { Carro com medicamento psicotrópico }\end{array}$ & $\begin{array}{l}06 \\
04 \\
02 \\
01 \\
01 \\
01\end{array}$ \\
\hline Subtotal & & 15 \\
\hline 3. Falha na comunicação & $\begin{array}{l}\text { - Entre equipe de enfermagem sobre mudança de leito } \\
\text { - Entre médico e equipe de enfermagem sobre medicação } \\
\text { suspensa, alteração da prescrição médica quanto à via de } \\
\text { administração } \\
\text { - Prescrição médica ilegível e por telefone }\end{array}$ & $\begin{array}{l}02 \\
02\end{array}$ \\
\hline Subtotal & & 10 \\
\hline 4. Falha no conhecimento & $\begin{array}{l}\text { - Conhecimento deficiente } \\
\text { - Cálculo errado do padrão de infusão intravascular }\end{array}$ & $\begin{array}{l}04 \\
02\end{array}$ \\
\hline Subtotal & & 06 \\
\hline Total & & 56 \\
\hline
\end{tabular}

As categorias serão discutidas separadamente. Falha no cumprimento de políticas e procedimentos

A categoria Falha no cumprimento de políticas e procedimentos, foi definida como "falhas no cumprimento de procedimentos para administração segura de medicamentos" ${ }^{(10)}$. Fuqua \& Stevens ${ }^{(10)}$ reconhecem que essa falha é um dos maiores fatores de erros na administração de medicamentos.

Nessa ótica, agrupamos um total de 25 relatos na categoria, subdivididos nas subcategorias: falha na execução da técnica (15) e na identificação do paciente(10).

Falha na execução da técnica foi definida como a falha do profissional de enfermagem em não executar corretamente o procedimento durante o preparo e administração do medicamento. Correspondem à falha os seguintes relatos: falha na leitura da dose medicamentosa durante o preparo do medicamento (06); na observação da punção venosa em quimioterapia (04); falha em não conferir a via de administração com a prescrição médica (01); falha em não conferir o medicamento disponível com a prescrição médica (01); em não identificar a medicação preparada (01); em não circular o horário da medicação de um paciente diabético em jejum (01) e no controle do gotejamento de solução intravascular (01). Os relatos abaixo podem ilustrar essas situações:

“...fui fazer a medicação das 14 hs e não tinha nada assim, eu não estava nervosa, eu não sei como eu enxerguei 1 1/2 cp, foi mesmo falta de atenção..."(Е.13).

“... eu não li, não percebi que era via oral, nem pelo cartão nem na prescrição que estava 1 cp de aminofilina de 8/8hs..." (E.08). 
“... não identificou a seringa, o nome da medicação, o número do leito, o nome do paciente, não anotou a via de administração, se tivesse feito teria evitado este erro..." (E.21).

Temos conhecimento de que, para assegurar uma administração de medicamento correta, ausente de falhas, algumas estratégias são utilizadas e observadas como os cinco "certos": medicamento certo, paciente certo, dose certa, via de administração certa e horário certo. Mesmo assim, continuam ocorrendo muitos erros, cujas causas os profissionais desconhecem, conforme a percebida nesta fala "olha e não presta atenção, pensa que vê uma coisa e vê outra, a gente aprende no curso que tem que ler e reler o que vai dar" (E. 22).

Acreditamos que, em tais situações, as falhas nas "tendências de confirmação" podem justificar tais erros; entretanto, há de se considerar que, atrelado a esses fatores, está o número inadequado de funcionários e excesso de atividades diárias, ocasionando sobrecarga de trabalho e estresse, que contribuem para a geração do erro, aumentando, assim, a sua incidência.

Falha na identificação do paciente foi considerada como aqueles relatos em que não houve a identificação correta do paciente por: ausência de pulseira no antebraço do paciente, leitos não identificados e não perguntar o nome do paciente no momento da administração do medicamento.

Em relação à falha na identificação do paciente, os profissionais de enfermagem referiram, em quatro relatos, que não havia pulseira de identificação no paciente, os leitos não estavam identificados em três relatos e, em outros três, não perguntaram o nome do paciente, administrando o medicamento somente pelo número do leito.

Em relação ao uso da pulseira de identificação, no hospital em foco, os profissionais de enfermagem referiram que não é colocada no paciente nem no momento e nem após a sua admissão no hospital, uma vez que essa prática somente é observada quando o paciente vai ser submetido a algum procedimento cirúrgico.

“...nós colocamos pulseiras de identificação na clínica somente quando o paciente vai para a cirurgia ..." (E.1).

“... os leitos não estavam identificados porque eram dois leitos e não é necessário, os pacien- tes também não tinham pulseira de identificação porque só usa quando vai para a cirurgia"(E.30).

Em estudo conduzido anteriormente por Miasso $\&$ Cassiani ${ }^{(11)}$, identificou-se que somente $6,8 \%$ dos pacientes hospitalizados faziam uso das pulseiras de identificação. Deles, somente $3 \%$ colocaram a pulseira após a internação, $21 \%$ já usaram a pulseira como preparo cirúrgico e $20,3 \%$ nunca fizeram uso da pulseira. Verificou-se, assim, que 76,2\% dos pacientes não estavam identificados com a pulseira no antebraço; o que é um dado alarmante face às dificuldades de identificação correta do paciente.

Como podemos, enquanto profissionais de enfermagem, assistir aos pacientes sob nossos cuidados e identificar o "paciente certo" se ele não faz uso da pulseira de identificação, e o seu leito não está identificado ? Podemos até depender do questionamento verbal, perguntando o seu nome, o que também pode conduzir ao erro, quando não se entende o nome corretamente ou quando pacientes são mentalmente confusos.

O método mais seguro ainda é o uso da pulseira para a identificação do paciente, ressalvando que pacientes com deficiência de audição e lesões cerebrais são pessoas que podem responder incorretamente acerca do nome. Assim, não checar a pulseira ou o nome do paciente é uma falha no cumprimento de políticas e procedimentos ${ }^{(10)}$.

Através dos relatos obtidos, percebemos dificuldades na identificação do paciente, seja através do antebraço ou do leito que ele ocupa. Há a necessidade do cumprimento de normas ou procedimentos, adotados pelo sistema, para evitar a troca de medicamentos no momento de atender os pacientes.

Entendemos que as instituições hospitalares, através de adequação dos recursos humanos disponíveis em número suficiente, qualificação profissional com educação contínua e procura de uma supervisão junto ao auxiliar de enfermagem, fornecerão condições essenciais para que ações de enfermagem sejam desenvolvidas com qualidade, sem acarretar danos ou prejuízos aos pacientes e prevenir o erro na administração de medicamentos.

Falha no sistema de distribuição e preparo dos medicamentos pela farmácia.

$\mathrm{Na}$ categoria Falha no sistema de distribuição e preparo dos medicamentos pela farmácia foram considerados os relatos relacionados às falhas no sistema de distribuição e preparo dos medicamentos pela farmácia. 
- Medicamento enviado com apresentação errada.

“... era uma medicação endovenosa que veio da farmácia, era para ser feita via oral, mas como veio em ampolas, eu fiz por via endovenosa..." (E.8).

- Atraso no horário de envio do carro de medicação à clínica.

"...o carro chegou às 18 horas, transbordando de medicação para conferir, é parenteral, é fungison, a gente tem que conferir item por item com o cartão, tem que checar, vem comprimido misturado, leva no mínimo $11 / 2$ hora para dar uma conferida e na medicação a gente fica sozinho, isso mexe com a cabeça de qualquer um, daí o motivo da pessoa acabar cometendo alguma coi$s a "$ (E.11).

- Muitos medicamentos no mesmo horário com atraso na administração

“... por ser um só e muito paciente, ninguém toma medicação na hora certa, um só toma, uma meia dúzia no horário os demais não, as insulinas eu faço primeiro porque senão eu vou chegar na enfermaria do paciente diabético uma hora e $1 / 2$ depois, dizem que é a melhor maneira de administrar medicamentos, apesar de dar a medicação adiantado ou atrasado para uns e uma meia dúzia no horário" (E.19).

Uma das maiores frequiências de relato (06) nesta categoria abordou o atraso no horário do envio do carro de medicação pela farmácia, o que interferiu na administração do medicamento ao paciente no horário certo, devido ao pouco tempo disponível para a conferência e administração dos medicamentos.

Discussões entre enfermeiros e farmacêuticos a respeito do atraso no horário de envio do carro de medicamentos, assim como a possibilidade de implantação do sistema de distribuição por dose unitária, devem ser realizadas a fim de evitar as situações mencionadas.

O sistema de dose única, ao contrário do utilizado nesse local de estudo, é usado em aproximadamente 90\% dos hospitais americanos. Dentre as vantagens mencionadas no sistema de dose única, estão a redução da frequiência dos erros de medicação, redução dos custos dos medicamentos, aumento no controle e uso do medicamento pela farmácia ${ }^{(12)}$.

Bradburry et al. ${ }^{(13)}$ afirmam que "a freqüência dos erros de medicação varia entre $10 \%$ e $20 \%$ no sistema tradicional comparada a $2 \%$ a $5 \%$ no sistema de distribuição por dose única".

Medidas administrativas devem ser tomadas também com respeito ao atraso no horário de envio do carro à unidade de enfermagem, que foi a situação que mais levou à ocorrência de erros dentro da categoria Falha no sistema de distribuição e preparo dos medicamentos pela farmácia.

\section{Falha na comunicação.}

Neste estudo há um total de 10 relatos agrupados na categoria Falha na comunicação. A definição desta categoria foi considerada neste trabalho e também por Fuqua \& Stevens ${ }^{(10)}$ como as falhas que envolveram a leitura, a audição e documentações incorretas, tais como caligrafia ruim, prescrições médicas verbais ou transcrições incorretas. As autoras acima citadas salientam que as falhas na comunicação são responsáveis por $39 \%$ dos erros registrados em um hospital.

Entendemos que essas situações foram derivadas da falta de integração e articulação entre a equipe multiprofissional envolvida na administração dos medicamentos. Observe as falas ilustrativas da situação:

“...o médico suspendeu a aminofilina em bomba de infusão e não sei o que aconteceu, ele não comunicou e passou para comprimido" (E.29).

“...foi falta de entendimento da prescrição médica, não era uma letra legível e os nomes eram parecidos, confundi amicacina com ampicilina..." (E.2).

A comunicação e a documentação de mudanças de leitos existentes no decorrer do plantão pelo enfermeiro são de extrema importância para o profissional de enfermagem que está responsável pelo carro de medicação visto que, muitas vezes, ele se baseia, erroneamente, somente no cartão de medicamentos para administrar os medicamentos, ao invés de verificar a prescrição médica.

Notamos que a letra ruim, ilegível ou mal escrita, associada aos nome de medicamentos semelhantes podem conduzir os profissionais de enfermagem ao erro. Segundo Fuqua \& Stevens ${ }^{(10)}$, "uma das fontes potenciais para o erro ocorrem quando prescrições ilegíveis são combinadas com o uso de numerosos medicamentos cujos nomes são semelhantes quanto ao som".

Enfermeiros e farmacêuticos não devem tentar decifrar caligrafias ruins ou ilegíveis, e sim, consultar 
o médico, quando possível, visando a esclarecimentos sobre a prescrição, o que deve ser estimulado, assim como, o uso de prescrições eletrônicas permitirá uma comunicação livre de interferências, dúvidas e más interpretações. Prescrições médicas ilegíveis são, portanto, consideradas situações de risco para a ocorrência de erros na medicação.

\section{Falha no conhecimento}

A categoria Falha no conhecimento foi definida como aquelas falhas que envolveram o despreparo teórico e prático dos profissionais de enfermagem a respeito da administração dos medicamentos e de suas propriedades farmacológicas.

“... fez a medicação psicotrópica pensando que era a outra, não conhecia, não sabia o nome da medicação, para que servia..." (E.4).

“... ela não sabia o que estava dando, que era um quimioterápico, não conhecia os princípios farmacológicos....ela não tinha a menor noção do risco que ela oferecia de um erro pela falta de conhecimento..." (Е.5).

“... o médico prescreveu leucovorin, que vem como ácido folínico, por causa do nome da medicação, a funcionária não tinha conhecimento que era o mesmo nome, não sabia que ácido folínico era a mesma coisa..."(Е.27).

A falta de preparo teórico para subsidiar a implementação segura da terapia medicamentosa constituiu-se em situações de risco para o paciente, atribuídas a uma falha no conhecimento farmacológico, cálculos errados de infusões intravasculares do medicamento e à falta de uma supervisão direta da tarefa pelo enfermeiro.

Visto que o sucesso na terapia medicamentosa depende do conhecimento do diagnóstico do paciente e das indicações do medicamento, como podemos, enquanto enfermeiros, delegar essa responsabilidade a profissionais de preparo educacional limitado sem uma supervisão direta da tarefa, como ocorre com freqüência na maioria das instituições?

Pierin et al. ${ }^{(14)}$ concluíram em seu estudo que uma das causas de iatrogenia em enfermagem é a deficiência do conhecimento teórico e prático do enfermeiro em relação a cálculos de gotejamento de soro, diluição, concentração e preparo da dosagem medicamentosa prescrita.

Os profissionais de enfermagem são os executores; estão na ponta final do processo da administra- ção de medicamentos, tendo, portanto, que conhecer os medicamentos a serem administrados aos pacientes sob sua responsabilidade para não colocar o paciente em situações de risco.

National Coordinating Council for Medication Error Reporting and Prevention-(NCCMERP) ${ }^{(5)}$ recomenda que os profissionais envolvidos na administração direta do medicamento devem conhecer:

- indicações e contra-indicações do uso da medicação;

- o resultado ou efeito esperado do medicamento administrado e as precauções, pois caso ocorra a manifestação de uma reação inesperada com o uso do medicamento, ele deve ser capaz de reconhecer as reações adversas;

- conhecer as possíveis interações que ocorrem com outras medicações ou alimentos, a fim de que ações sejam tomadas rapidamente com o paciente.

Há a necessidade de um serviço de educação continuada atuante, no que diz respeito à administração do medicamento, a fim de proporcionar novos conhecimentos aos profissionais de enfermagem, dando-lhes a oportunidade de atualizarem-se, ou até de reciclarem os conhecimentos adquiridos, aprimorando-os.

Entre as várias condutas a serem tomadas para prevenir os erros de medicação, "a mais importante, sem dúvida, é a educação, não se limitando somente à educação em serviço, função da instituição empregadora, mas também àquela relativa à formação profissional" (15).

Para que haja uma diminuição da ocorrência de erros na medicação relativas às falhas no conhecimento, torna-se imprescindível uma avaliação periódica dos funcionários sob supervisão do enfermeiro, a fim de detectar as dificuldades encontradas no momento do preparo e administração do medicamento.

\section{5- CONCLUSÕES}

Este estudo identificou quatro categorias de situações de riscos que conduziram à ocorrência de erros na administração de medicamentos: falha no cumprimento de políticas e procedimentos, falha no sistema de distribuição e preparo dos medicamentos pela farmácia, falha na comunicação e falha no conhecimento.

$\mathrm{Na}$ categoria falha no cumprimento de políticas e procedimentos, há a necessidade do cumprimento e revisão dos cinco "certos" no preparo e 
administração do medicamento, principalmente, a identificação do paciente através do uso da pulseira no antebraço, no leito ou perguntar o seu nome, assim como a verificação da dose, do medicamento, do horário e via de administração.

Fornecer um ambiente seguro para a administração de medicamentos envolve um grande número de recursos, tanto físicos (luminosidade, controle de temperatura, presença de ruídos, interrupções pessoais ou por telefone) como humanos (aquisição de conhecimentos e anos de experiência). A adequação dos recursos humanos e carga de trabalho pareceu-nos fundamental para que ocorra uma prática segura na administração de medicamentos.

$\mathrm{Na}$ categoria falha no sistema de distribuição e preparo dos medicamentos pela farmácia, para minimizar tais falhas são propostas novas formas de distribuição do medicamento, como a implantação do sistema de dose única.

Com o objetivo de melhorar a situação - falha na comunicação - entre a equipe multiprofissional, recomenda-se a automatização do sistema para promover uma comunicação rápida, segura e atualizada sobre alterações da prescrição médica e mudanças na terapia medicamentosa do paciente.

Em relação à categoria falha no conhecimento, há evidências da necessidade de educação contínua e reciclagem profissional pelo enfermeiro e sua equipe, no que concerne à atualização de conhecimentos acerca da administração de medicamentos, como preconiza o Código de Ética dos Profissionais de En- fermagem, no capítulo III - Das responsabilidades, no artigo 18, consta: "manter-se atualizado ampliando seus conhecimentos técnicos, científicos e culturais, em benefício da clientela, coletividade e do desenvolvimento da profissão”; no capítulo V- Das Proibições, artigo 47: "administrar medicamentos sem certificar-se da natureza das drogas que o compõem e da existência de risco para o cliente"(16).

As inovações tecnológicas, o avanço rápido e crescente do mercado farmacêutico transformam a nossa realidade profissional intensamente; novos conhecimentos ou atualizações contínuas são primordiais para a manutenção no campo da atividade profissional, principalmente para os que atuam na área da saúde.

Ressalta-se a necessidade de atualizações, aprimoramento ou de reciclagem para atualização de conhecimentos adquiridos na formação básica curricular e assumir o aumento proporcional da responsabilidade ética inerente aos profissionais da área da saúde.

Assim, neste estudo, identificamos as situações que conduziram os profissionais de enfermagem aos erros na medicação, entretanto maiores investigações de suas causas, índices e conseqüências devem ser motivadas pelas instituições hospitalares, pois os erros de medicação são indicadores de qualidade de assistência.

\section{AGRADECIMENTOS}

À FAPESP- Fundação de Amparo à Pesquisa do Estado de São Paulo pelo recurso financeiro concedido.

CARVALHO VT \& CASSIANI SHB. Medication errors: analysis of situations reported by nursing staff. Medicina, Ribeirão Preto, 33: 322-330, july/sept. 2000.

ABSTRACT: Medication errors are one indicator of the quality of the health care provided to hospitalized patients. The purpose of this study was to analyze the situations of errors in medications related by nursing staff. The study was developed at the interior of the state of São Paulo, Brazil. Data were collected through interviews with 7 nurses, 1 nursing technician and 23 nursing auxiliaries from the medical clinic of the mentioned hospital. The author adopted as the methodological reference adaptation of the critical incident technique. The analysis enabled the identification of fifty six (56) situations grouped into four categories: failure in the accomplishment of policies and procedures (25), failure in the system of medications distribution and preparation by the pharmacy (15), communication failure (10) and knowledge failure (06). Thus, a safe environment with availability of human and physical resources is necessary for the prevention of future medication errors, as well as investments to provide knowledge on medication administration to nursing professional, aiming at a nursing care with quality.

UNITERMS: Medication Errors. Drugs. Nursing. 


\section{REFERÊNCIAS BIBLIOGRÁFICAS}

1 - TAKAKURA MS. Avaliação morfométrica do efeito da massagem e ou calor no músculo gluteus-máximus e ratos, Tese de Doutorado, Escola de Enfermagem de Ribeirão Preto da USP, Ribeirão Preto, p.1-109, 1988.

2 - ARCURI EAM. Reflexões sobre a responsabilidade do enfermeiro na administração de medicamentos. Rev Escola Enf USP 25: 229-237, 1991.

3 - CASSIANI SHB \& OLIVEIRA VT. Análise técnica e científica da administração de medicamentos por via intramuscular em crianças por auxiliares de enfermagem. Acta Paul Enf 10: 49-61, 1997.

4 - CASSIANI SHB; RANGEL SM \& TIAGO F. Complicações após aplicações por via intramuscular do diclofenaco de sódio: estudo de caso. Medicina, Ribeirão Preto, 31: 99-105,1998.

5 - NATIONAL COORDINATING COUNCIL FOR MEDICATION ERROR REPORTING AND PREVENTION. NCC MERP taxonomy of medication errors. Rockville: NCC MERP, 1998. [13.11.1998]. Disponível em URL:_http://www.nccmerp.org/ taxo 0514.pdf.

6 - BATES DW; CULLEN DJ \& LAIRD N. Incidents of adverse drugs events and potential adverse drug events: implications for prevention. JAMA 274: 29-34, 1995.

7 - WOLF ZR; MCGOLDRICK MSN; FLYNN ER \& WARWICK BSN Factors associated with a perceived harmful outcome from medication errors: a pilot sudy. J Cont Educ Nurs 27: 65-74, 1996.

8 - CARVALHO VT; CHIERICATO C; CASSIANI SHB \& MIASSO AI. Erros mais comuns e fatores de risco na administração de medicamentos em unidades básicas de saúde. Rev Latinoam Enf 7: 67-75,1999.
9 - FLANAGAN JC. A técnica do incidente crítico. Arq Bras Psicol Apl 21: 99-141, 1973.

10 - FUQUA RA \& STEVENS KR. What we know about medication errors: a literature review. J Nurs Qual Assur 3: 1-17, 1988.

11 - MIASSO AI \& CASSIANI SHB. Identificação do paciente como fator minimizador dos erros na administração de medicamentos. Simpósio de Iniciação Científica da USP 6 : 78,1998 .

12 - MCNALLY KM; PAGE MA \& SUNDERLAND BY. Failuremode and effect analysis in improving a drug distribution system. Am J Healthy Syst Pharm 54: 171-177, 1997.

13 - BRADBURRY K; WANG J; HASKINS G \& MEHL B. Prevention of medication errors: developing a continuous- quality- improvement approach. Mt Sinai J Med 60: 379-386, 1993.

14 - PIERIN A; IDE CAC; PADILHA KG \& MARKIEVICZ W. latrogenia em enfermagem. Rev Esc Enf USP 17: 11925,1983 .

15 - CASSIANI SH.B. Um salto para o futuro no ensino da administração de medicamento: desenvolvimento de um programa instrucional auxiliado pelo computador, Tese de Livre Docência, Escola de Enfermagem de Ribeirão Preto da USP, Ribeirão Preto, p. 1-206,1998.

16 - CONSELHO REGIONAL DE ENFERMAGEM. Documentos básicos de Enfermagem. São Paulo, 1998.

Recebido para publicação em 10/12/1999

Aprovado para publicação em 11/07/2000 